Poly(3,4-ethylenedioxythiophene) (PEDOT) polymer coatings facilitate smaller neural recording electrodes

This article has been downloaded from IOPscience. Please scroll down to see the full text article.

2011 J. Neural Eng. 8014001

(http://iopscience.iop.org/1741-2552/8/1/014001)

View the table of contents for this issue, or go to the journal homepage for more

Download details:

IP Address: 141.211.173.82

The article was downloaded on 06/04/2012 at 16:55

Please note that terms and conditions apply. 


\title{
Poly(3,4-ethylenedioxythiophene) (PEDOT) polymer coatings facilitate smaller neural recording electrodes
}

\author{
Kip A Ludwig ${ }^{1,3}$, Nicholas B Langhals ${ }^{1}$, Mike D Joseph ${ }^{1}$, \\ Sarah M Richardson-Burns ${ }^{2}$, Jeffrey L Hendricks ${ }^{2}$ and Daryl R Kipke ${ }^{1}$ \\ ${ }^{1}$ Department of Biomedical Engineering, University of Michigan, Ann Arbor, MI 48109, USA \\ 2 Biotectix, LCC, Ann Arbor, MI 48109, USA \\ E-mail: ludwigk@umich.edu and dkipke@umich.edu
}

Received 22 October 2010

Accepted for publication 25 November 2010

Published 19 January 2011

Online at stacks.iop.org/JNE/8/014001

\begin{abstract}
We investigated using poly(3,4-ethylenedioxythiophene) (PEDOT) to lower the impedance of small, gold recording electrodes with initial impedances outside of the effective recording range. Smaller electrode sites enable more densely packed arrays, increasing the number of input and output channels to and from the brain. Moreover, smaller electrode sizes promote smaller probe designs; decreasing the dimensions of the implanted probe has been demonstrated to decrease the inherent immune response, a known contributor to the failure of long-term implants. As expected, chronically implanted control electrodes were unable to record well-isolated unit activity, primarily as a result of a dramatically increased noise floor. Conversely, electrodes coated with PEDOT consistently recorded high-quality neural activity, and exhibited a much lower noise floor than controls. These results demonstrate that PEDOT coatings enable electrode designs $15 \mu \mathrm{m}$ in diameter.
\end{abstract}

(Some figures in this article are in colour only in the electronic version)

\section{Introduction}

The utility of implantable microelectrode arrays is currently limited by the size of the individual electrode sites for a number of reasons. First, smaller electrode sites facilitate the development of more densely packed microelectrode arrays. At present, researchers are limited to sampling from a few neurons out of the billions of neurons which execute function in the brain [1]. As a result, the study of how large networks of neurons interact to produce biologically relevant behaviors is severely hampered. Similarly, the efficacy of modern neuroprosthetic devices is greatly dependent upon the observable number of neural inputs/outputs. Second, smaller electrode sites promote the design of smaller arrays, which in turn cause less damage upon implantation. Recent studies

\footnotetext{
3 Author to whom any correspondence should be addressed.
}

indicate that probe dimensions smaller than $12 \mu \mathrm{m}$ minimize the reactive cell responses that negatively impact long-term neural recordings [2-4].

Unfortunately, decreasing the size of an electrode site increases the impedance, which can degrade recordings. Impedance impacts recordings primarily through two mechanisms: noise and shunt loss [5]. Noise at the electrode/ electrolyte interface can arise from random fluctuations of charged carriers-either electrons in an electrical conductor (known as Johnson or thermal noise), or ions in an electrolytic medium [6-10]. These fluctuations can be caused by Brownian motion of electrons, drift and diffusion of charged ions due to concentration gradients, oxidation/reduction reactions occurring at the electrode/electrolyte interface, etc [10]. As these random movements occur they create current perturbations, which increase the voltage noise in proportion 
to impedance [10]. Noise resulting from the fluctuation of charged particles (which we will refer to as fluctuation noise hereafter) represents only one source of noise out of many possible sources: instrumentation noise, shot noise, flicker noise, biological noise, etc. Sources of noise sum in quadrature $\left(a^{2}+b^{2}=c^{2}\right)$, meaning that the single largest source of noise tends to dominate the noise floor. Functionally, this means that the fluctuation noise for an electrode will only contribute marginally to the total noise floor until a nominal impedance magnitude is reached. Beyond this impedance magnitude, fluctuation noise will tend to dominate the observed noise floor.

Shunt loss is defined as the loss of signal from the electrode and measurement system to ground. This can be separated into three distinct circuit elements. First, there is a capacitive loss from the metal traces on the microelectrode to the surrounding cerebral spinal fluid (CSF) [9]. Second, a resistive element models the loss of signal from the inherent resistive nature of the metal wiring from the electrode to the measurement system [9]. Finally, there is a capacitor that models the capacitive signal loss in the measurement system [9]. Like fluctuation noise, shunt loss increases in proportion to impedance [9]. Typically, electrodes with impedances of $5 \mathrm{M} \Omega$ or greater have levels of fluctuation noise and shunt loss that make recording from individual neurons problematic $[5,9,11]$.

Conductive polymer coatings have been hypothesized to be an enabling technology for smaller electrode designs [12-17]. Conductive polymers increase the electrochemical surface area (ESA) of an electrode without changing its geometric surface area (GSA: e.g. diameter, circumference), lowering impedance [18-21]. In 2006, the Kipke lab demonstrated that surfactant-templated poly $(3,4-$ ethylenedioxythiophene) (PEDOT) could be used to improve chronic neural recordings from standard-sized electrodes [12].

Building on this earlier work, in this paper we demonstrate that PEDOT coatings effectively lower the impedance of small, gold recording electrodes $(15 \mu \mathrm{m}$ in diameter) with initial impedances outside of the effective range. Chronically implanted unmodified electrodes were unable to record wellisolated unit activity, primarily because of an increased noise floor arising from large site impedances. Conversely, sites coated with PEDOT were able to consistently record highquality neural activity, and exhibited a markedly lower noise floor than controls.

\section{Methods}

\subsection{Microelectrodes}

Three male Sprague-Dawley rats were implanted with three 16-channel chronic silicon 'Michigan' microelectrode arrays, using experimental procedures outlined previously [12, 22]. Arrays consisted of four shanks, each with four evenly spaced gold electrodes. Site and shank separations were sufficient (100 $\mu \mathrm{m}$ or greater) to limit the probability of an individual neuron being recorded from multiple sites [23]. All electrodes used in this study were $15 \mu \mathrm{m}$ in diameter, or a GSA of $177 \mu \mathrm{m}^{2}$.

\subsection{Electrochemical deposition and initial evaluation}

Electrochemical deposition of PEDOT in this study was accomplished using an electrochemical potentiostat/ galvanostat (Autolab PGSTAT12, Eco Chemie, Urtecht, The Netherlands) with associated General Purpose Electrochemical System (GPES) software. PEDOT doped with tetraethylammonium perchlorate and dissolved in $20 \mathrm{wt} \%$ surfactant poly(oxyethylene)10-oleyl ether was galvanostatically deposited onto the gold sites of the neural probes [24]. Based on results from our prior paper, PEDOT films generated with a deposition charge of $260 \mathrm{mC} \mathrm{cm}^{-2}$ were chosen for in vivo testing. Scanning electron microscopy (SEM) demonstrated that the PEDOT film deposited using a charge of $260 \mathrm{mC} \mathrm{cm} \mathrm{cm}^{-2}$ did not increase the diameter of the recording site (figure 1). Consequently, at $260 \mathrm{mC}$ $\mathrm{cm}^{-2}$ the PEDOT film increased the ESA of the electrode without increasing the GSA. Eight sites on each probe were deposited with surfactant-templated ordered PEDOT film. The deposited sites were staggered in relative location to prevent bias due to specific shank location or cortical depth (figure 3). The remaining eight sites on each probe were left uncoated as controls for comparison.

\subsection{Surgical techniques}

All of the arrays in this study were implanted in motor cortex, targeting cortical layer $V$, as outlined in previous work [12, 22, 25]. Initial anesthesia was administered via intra-peritoneal injections of a mixture of $50 \mathrm{mg} \mathrm{ml}^{-1}$ ketamine, $5 \mathrm{mg} \mathrm{ml}^{-1}$ xylazine and $1 \mathrm{mg} \mathrm{ml}^{-1}$ acepromazine at an injection volume of $0.125 \mathrm{ml} / 100 \mathrm{~g}$ body weight. Updates of $0.1 \mathrm{ml}$ ketamine $\left(50 \mathrm{mg} \mathrm{ml}^{-1}\right)$ were delivered as needed to maintain anesthesia during the surgery. Animals were secured to a standard stereotaxic frame, and three stainless steel bone-screws were inserted into the skull. The electrode connector was grounded to a bone-screw over parietal cortex using a stainless steel wire.

A craniotomy approximately $3 \mathrm{~mm}$ by $2 \mathrm{~mm}$ was made over the target area (target location $3.0 \mathrm{~mm}$ anterior to bregma, $2.5 \mathrm{~mm}$ lateral from bregma and $1.4 \mathrm{~mm}$ deep from the surface of the brain). Two incisions were made in the dura mater to create four flaps, which were subsequently folded back over the edge of the craniotomy. The electrodes were then hand-inserted using microforceps into the approximate target cortical area. Cortical depth was estimated using the known location of the electrode sites on the individual shanks in conjunction with the known length of the individual shanks. Next, the surface of the brain was covered with GelFoam ${ }^{\circledR}$ (Henry Schein, Inc., Miami, FL, USA) for protection. The silicon cable connector was covered with either remaining GelFoam or Kwik-Sil silicone polymer (World Precision Instruments, Inc., Sarasota, FL, USA). The entire assembly excluding the connector was then enclosed using dental acrylic (Co-Oral-Ite, Dental Mfg. Co., Santa Monica, CA, USA). Finally, sutures were used to close the skin around the acrylic, and triple-antibiotic ointment was applied. All procedures complied with the United States Department of Agriculture guidelines for the care and use of laboratory animals and were approved by the University of Michigan Animal Care and Use Committee. 
$(A)$

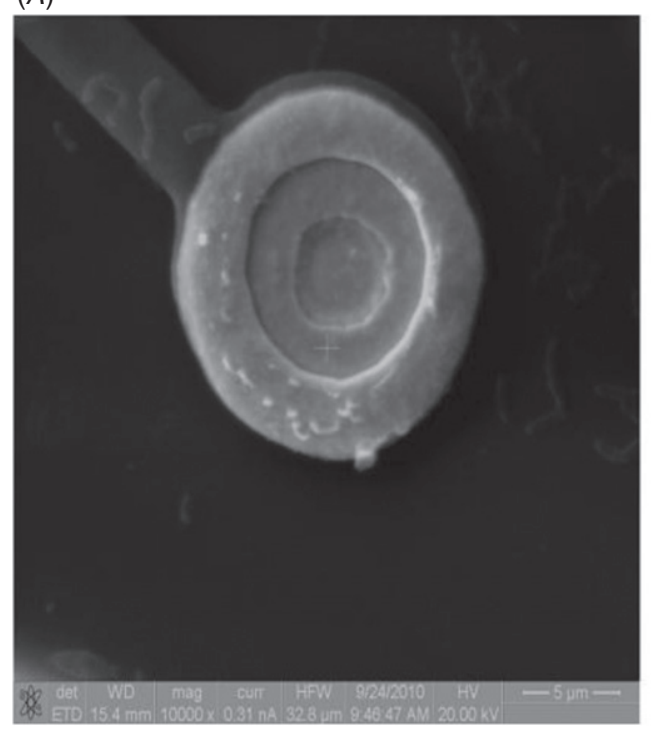

$(B)$

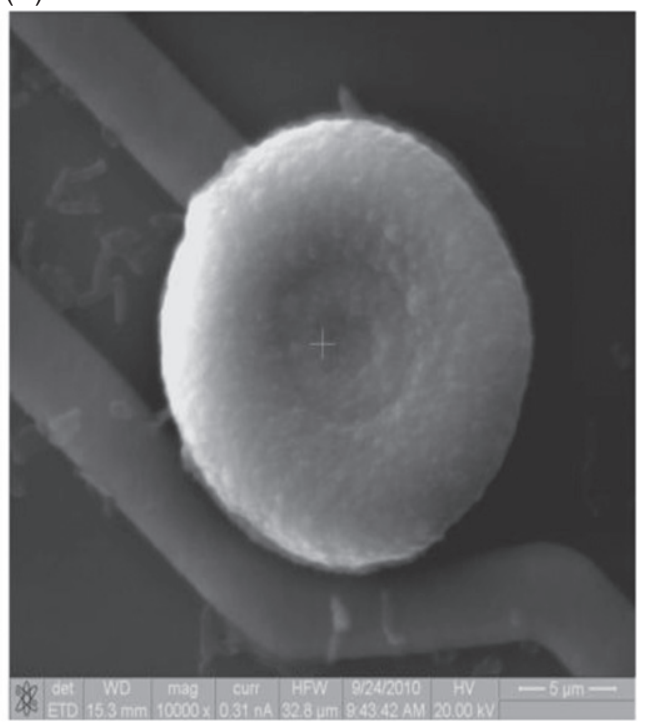

Figure 1. Scanning electron microscopy, PEDOT coatings. (A) A PEDOT film generated using a deposition charge of approximately $260 \mathrm{mC} \mathrm{cm}^{-2}$, and (B) a PEDOT film generated using a deposition charge of approximately $1600 \mathrm{mC} \mathrm{cm}^{-2}$. Note that the PEDOT film generated using $260 \mathrm{mC} \mathrm{cm}^{-2}$ does not increase the geometric diameter of the underlying gold site, whereas the film generated using a deposition charge of $1600 \mathrm{mC} \mathrm{cm}^{-2}$ increases the effective diameter of the gold site by approximately $20 \%$. For this study, PEDOT films were generated using a deposition charge of $260 \mathrm{mC} \mathrm{cm}^{-2}$.

\subsection{Neural recordings and data analysis}

Recorded neural signals were acquired using a Plexon multichannel neural acquisition processor (MNAP; Plexon Inc., Dallas, TX, USA). Neural electrophysiological recordings for all 16 channels were amplified and bandpass filtered; single and multi-unit recordings were sampled at $40 \mathrm{kHz}$ (Plexon) and bandpass filtered from 450 to $5000 \mathrm{~Hz}$. All recordings were taken in reference to a distant stainless steel (316-SS grade) bone screw inserted through the skull during surgery. During recording sessions, animals were placed in an electrically shielded recording booth and multiple $30 \mathrm{~s}$ segments of continuous neural recordings were taken. Animals were lightly anesthetized with ketamine/xylazine throughout the data collection sessions.

Neural recording segments were analyzed offline to determine the number of neurons recorded, noise levels and signal amplitudes using custom-automated MATLAB (Mathworks Inc., MA, USA) software, as described in detail [12] and utilized elsewhere [12, 21, 25-29]. In summary, an amplitude threshold window was set 3.5 standard deviations above and below the mean of the sample distribution. For each peak exceeding the threshold window, a $2.4 \mathrm{~ms}$ candidate waveform snippet centered on the absolute minimum of the waveform was removed from the recorded segment and stored. The amplitude of the noise voltage for every recording site in each recorded segment was calculated after all candidate waveforms had been removed.

After initial principal component analysis and fuzzy Cmeans clustering [12], waveforms with a cluster membership index of greater than 0.8 were used to determine a mean waveform for a cluster. An interspike interval histogram for each cluster was generated and visually inspected for an obvious absolute refractory period as an additional measure of noise rejection. Signal amplitude for a cluster was defined as the peak-to-peak amplitude of the mean waveform for each cluster.

The signal-to-noise ratio (SNR) for a given cluster was defined as follows:

$$
\begin{aligned}
\mathrm{SNR}= & \text { Signal amplitude } /(2 * \text { calculated } \mathrm{RMS} \text { noise } \\
& \text { voltage for recording site }) .
\end{aligned}
$$

Clusters were then separated into one of four categories based on calculated SNR. Clusters with an SNR of greater than 4 were categorized as quality units. Clusters with an SNR between 3 and 4 were categorized as moderate units. Clusters with an SNR between 2 and 3 were categorized as poor units, while clusters with an SNR of less than 2 were not considered units. These four categories correspond well with observations of unit quality based on SNR made in similar recording studies $[12,23,25]$.

Isolating action potentials from an individual neuron using an individual recording site is inherently prone to classification errors [30, 31]. The methodology employed in this study is intended to minimize these errors, and should accurately parallel the true number of underlying neural sources. The sorting routine produces similar results to manual sorting performed by experienced researchers over the same data sets, but with the advantage of being objective and automated [12].

\subsection{Impedance spectroscopy measurements}

Impedance spectroscopy measurements were made using an Autolab potentiostat PGSTAT12 (Eco Chemie, Utrecht, The Netherlands) with associated frequency response analyzer (Brinkmann, Westbury, NY, USA). Impedance measurements were made by applying a $25 \mathrm{mV}$ RMS sine wave with frequencies varied logarithmically from $10 \mathrm{~Hz}$ to $10 \mathrm{kHz}$. Prior to implantation, measurements were made by immersing 

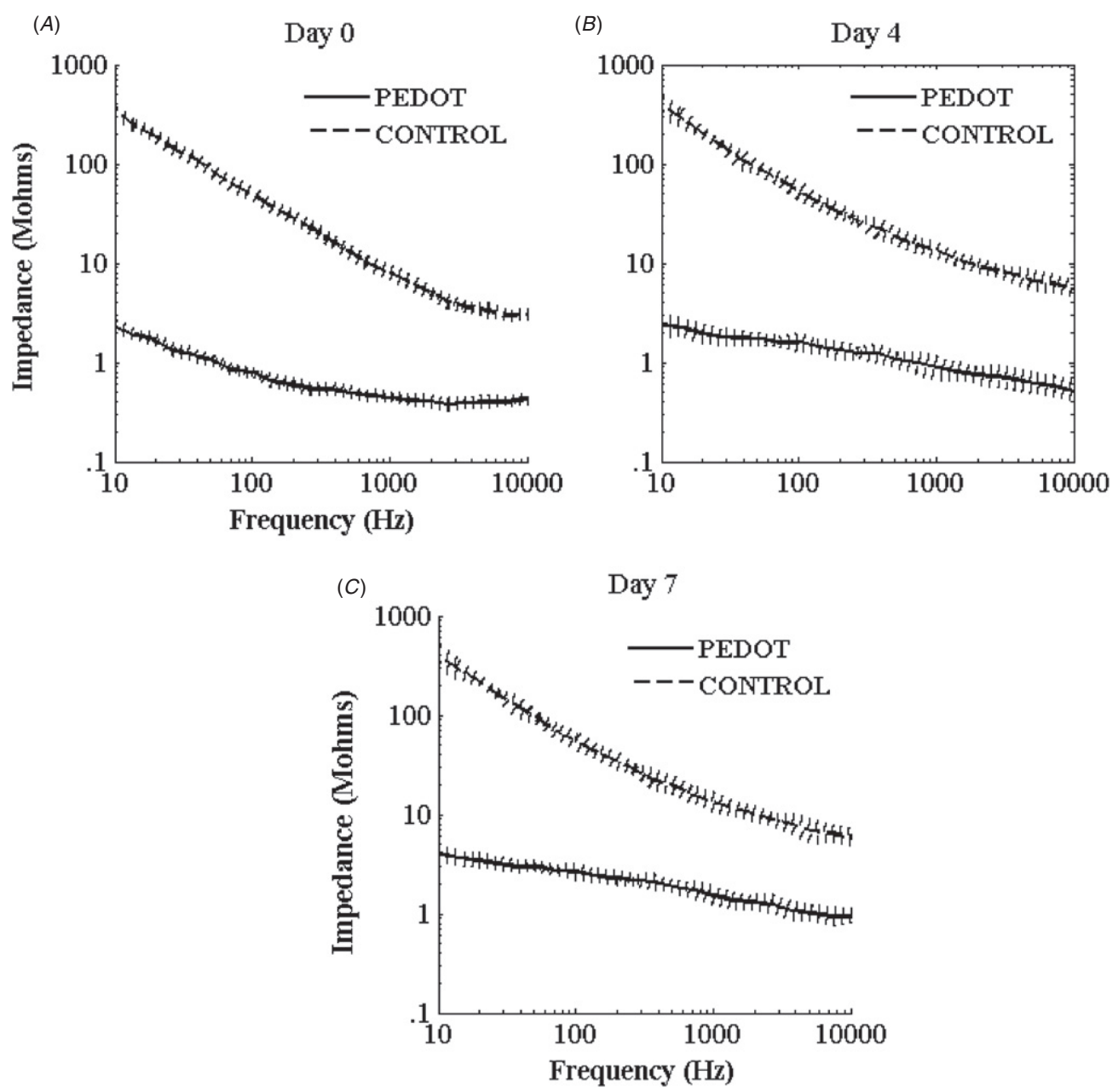

Figure 2. Bode plot of average measured impedance versus frequency. The dotted lines denote standard error of the data set on the given day $(n=24)$. (a) Day zero post-implantation. (b) Day four post-implantation. (c) Day seven post-implantation. As the immune response to the implant progresses during the first seven days after surgery, impedances at the critical $1 \mathrm{kHz}$ frequency increase for both PEDOT and control sites.

the electrode recording sites in $0.1 \mathrm{M}$ phosphate buffer saline (PBS), and a platinum foil was used as the reference electrode. After implantation, a distant stainless steel (316-SS grade) bone screw was used as the reference electrode.

\subsection{Statistical analysis}

For this study, comparative statistical significance between groups was determined using standard analysis of variance techniques (ANOVA). There were 24 PEDOT modified sites and 24 control sites on any specific day in the experiment. The factors used in initial comparative ANOVA calculations for any given metric were coated versus control, animal and day number. The associated calculated standard deviation has been included in the text with all average measurements.

\section{Results and discussion}

\subsection{Impedances}

The impedances of the electrodes at $1 \mathrm{kHz}$ were used for comparison purposes as action potentials have a characteristic frequency band centered at $1 \mathrm{kHz}$. Prior to implantation, the
$1 \mathrm{kHz}$ impedance of the unmodified gold sites ranged between 6 and $11 \mathrm{M} \Omega$, with a mean impedance of $9.1 \pm 1.4 \mathrm{M} \Omega$ (see table 1). After electrochemical deposition with PEDOT, in vitro site impedances ranged between 0.3 and $0.6 \mathrm{M} \Omega$, with a mean impedance of $0.37 \mathrm{M} \Omega \pm 0.05$.

Over the seven days following implantation, impedance increases were noted in both PEDOT and control sites that were consistent with trends observed in other microelectrode recording studies performed in the rat model [12, 21, 22, 25]. Figure 2 depicts Bode plots of the average impedance magnitude for PEDOT and control sites at day zero, day four, and day seven post-surgery. Over the first two days following implantation, the average impedance of the PEDOT sites at $1 \mathrm{kHz}$ increased slightly to $0.45 \pm$ $0.1 \mathrm{M} \Omega$, while the average impedance of the control sites also slightly increased to $9.2 \pm 1.7 \mathrm{M} \Omega(p<0.001$, see table 1). Between days three and five post-implantation, a more marked impedance increase at $1 \mathrm{kHz}$ was observed on both PEDOT and control sites to $1.31 \pm 0.4 \mathrm{M} \Omega$ and $10.3 \pm 1.9 \mathrm{M} \Omega$, respectively ( $p<0.001$, see table 1$)$. During the peak of the early reactive response, spanning from days six to eight, the mean impedance of PEDOT sites further increased to $2.21 \pm 0.7 \mathrm{M} \Omega$, while the mean impedance of the 

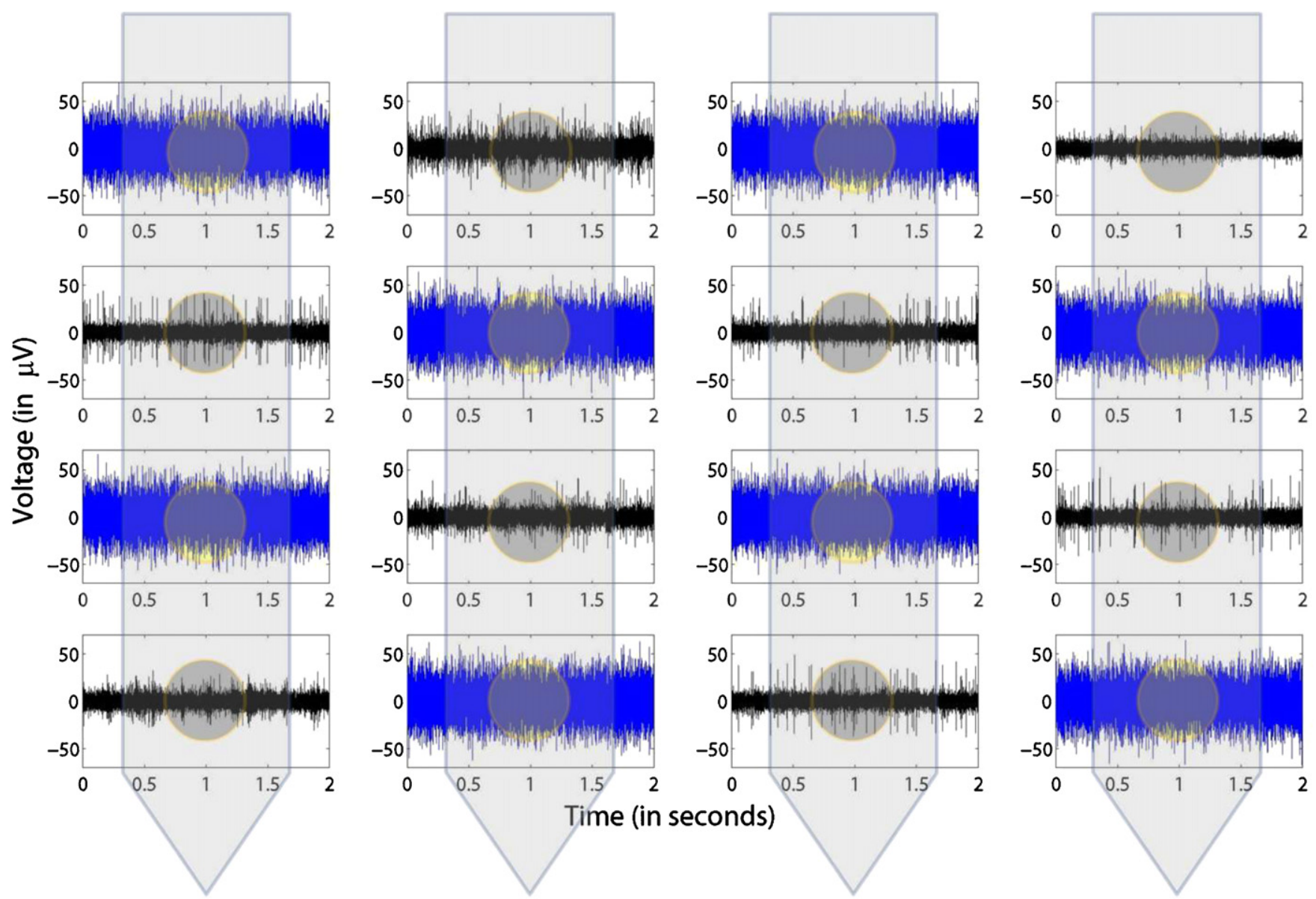

Figure 3. Representative high speed data across one four-shank array. Black traces are PEDOT sites, while gray (blue online) traces denote controls. The noise floor on control sites is dramatically larger than the noise floor on PEDOT sites, presumably obscuring unit activity.

Table 1. Summary of results for groups of days after surgery. PEDOT site averages are listed in black, while control site averages are listed in blue.

\begin{tabular}{|c|c|c|c|c|c|c|c|c|}
\hline & \multicolumn{2}{|c|}{$\begin{array}{l}\text { Average impedance } \\
(\mathrm{M} \Omega)\end{array}$} & \multicolumn{2}{|c|}{ Pk-Pk noise $(\mu \mathrm{V})$} & \multicolumn{2}{|c|}{ Signal amplitude $(\mu \mathrm{V})$} & \multicolumn{2}{|c|}{ Units per site } \\
\hline & PEDOT & Control & PEDOT & Control & PEDOT & Control & PEDOT & Control \\
\hline Days 0-2 & $0.45 \pm 0.1$ & $9.2 \pm 1.7$ & $35.3 \pm 5.3$ & $106.2 \pm 8.2$ & $55.2 \pm 20.2$ & N.A. & $0.8 \pm 0.1$ & 0 \\
\hline Days 3-5 & $1.31 \pm 0.4$ & $10.3 \pm 1.9$ & $46.2 \pm 8.1$ & $111.3 \pm 10.1$ & $84 \pm 14.4$ & N.A. & $0.5 \pm 0.2$ & 0 \\
\hline Days 6-8 & $2.21 \pm 0.7$ & $11.4 \pm 2.2$ & $51.3 \pm 9.3$ & $116.8 \pm 9.5$ & $95.3 \pm 17.1$ & N.A. & $0.4 \pm 0.2$ & 0 \\
\hline
\end{tabular}

unmodified sites also increased to $11.4 \pm 2.2 \mathrm{M} \Omega(p<0.001$ see table 1).

\subsection{Noise}

As anticipated, the average peak-to-peak noise levels over the time frame of this experiment were dramatically larger on control sites (Control mean: 106.2 $\pm 8.2 \mu \mathrm{V}$, PEDOT mean: $35.3 \pm 5.3 \mu \mathrm{V})$. Over the seven days following implantation, the trend in average peak-to-peak noise levels on both PEDOT and control sites paralleled their impedance trends (see table 1). In the period spanning days three and five postsurgery, the average peak-to-peak noise level on PEDOT sites increased to $46.2 \pm 8.1 \mu \mathrm{V}$, while control sites increased to $111.3 \pm 10.1 \mu \mathrm{V}$. During days six to eight post-surgery, the average peak-to-peak noise levels on PEDOT and control sites yet again increased to $51.3 \pm 9.3 \mu \mathrm{V}$ and $116.8 \pm 9.5 \mu \mathrm{V}$, respectively.

\subsection{Unit recordings}

Quality unit activity was evident on at least some PEDOT treated sites on all three arrays at every time point in this study (see table 1). The average number of recorded units for PEDOT sites was highest in the days immediately following surgery $(0.8 \pm 0.1$ average units per site; total number of quality units/total number of sites), and then diminished over the week following surgery $(0.4 \pm 0.2$ average units per site between days six and eight following surgery). A decreasing trend in unit activity in the week following surgery has been noted elsewhere $[12,25,26,32]$ and is hypothesized to be caused by edema/swelling coupled with the initial immune response. 


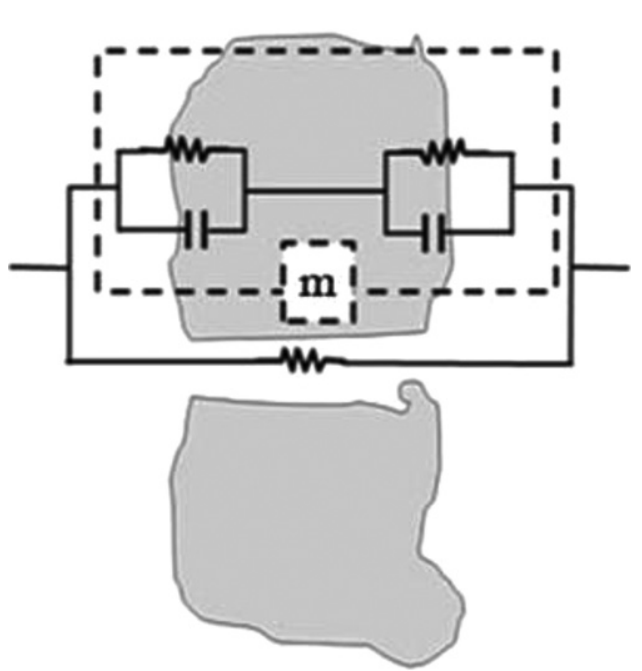

Figure 4. One individual element of the lumped circuit model for encapsulation.

In contrast to the PEDOT sites on the three implanted arrays, no quality unit activity was evident on the control sites at any point during this study (see table 1). Recordings on control sites were dominated by a large noise floor, which was sufficient to obscure all unit activity on these sites (see figure 3). The high initial impedance of the untreated sites resulted in a large fluctuation noise contribution, dramatically reducing the likelihood of observing neural activity sufficient in amplitude to be differentiated from the noise floor.

\subsection{Contribution of encapsulation}

Inserting a microelectrode into brain tissue elicits a reactive foreign body response, which produces a fibrous encapsulation of the array, effectively creating a high impedance barrier between the microelectrode and the neuron population [3335]. A well-described theoretical circuit model of this phenomenon has been widely accepted [36-39]. The tissue encapsulation of the array can be characterized by a sealing resistance, describing protein adsorption and in some cases a layer of connective tissue. In addition, the model incorporates adjacent cellular layers of glia and macrophages given by a membrane capacitance, a membrane resistance and a membrane area scaling term, $m$, related to encapsulation thickness and cell-to-cell adhesion within the cellular layer (see figure 4). The extracellular pathway between cells is defined as a resistance.

Based on this model, the chronic foreign body response can dramatically increase the effective overall impedance of a chronically implanted electrode. In previous studies describing the changes in electrode impedance of typically sized cortical microelectrodes following implant, the contribution of the encapsulation response to total electrode impedance became dominant after a few weeks [12, 21, 22, 26]. Consequently, increasing the pre-implant surface area using a conductive polymer coating provided limited chronic benefit to neural recordings [12].

In this study of small electrode sites $15 \mu \mathrm{m}$ in diameter, the obvious benefit of PEDOT coatings to neural recordings was still evident at eight days post-implant. This time point is important, as the contribution of encapsulation to electrode impedance typically reaches a maximum one to two weeks post-surgery $[12,21,28,40]$, demonstrating that PEDOT coatings enable neural recordings from small electrode sites even when the impedance contribution of encapsulation is at a maximum. These results indicate that as the size of the electrode decreases and total impedance approaches $5 \mathrm{M} \Omega$ and beyond, coatings to increase the surface area of the electrode become critical to minimize fluctuation noise and shunt loss.

Although the PEDOT films enabled recordings through 15 $\mu \mathrm{m}$ diameter sites, by day eight the average number of quality units per PEDOT site had diminished to 0.4 units per site, presumably due to the inherent foreign body response to the electrode. Consequently, future work will focus on utilizing PEDOT coatings to enable smaller multi-electrode arrays that cause less damage upon implantation. Recent studies have indicated that by reducing the size of the implanted electrode, the foreign-body response to the electrode can be minimized, decreasing the contribution of encapsulation to impedance [3, 4, 41, 42] and possibly limiting neuronal death in the vicinity of the electrode array [43]. Although PEDOT is one enabling component for manufacturing smaller multielectrode arrays, a number of technical problems need to be addressed, e.g. insulation requirements, interconnect reliability, crosstalk between adjacent traces, before these arrays become a practical reality.

\section{Conclusions}

The next generation of neural electrodes needs to facilitate more channels of communication to and from the brain over a smaller tissue area. Moreover, these electrodes need to be smaller in order to limit neural damage upon implantation and long-term insult to the surrounding tissue. In this study, PEDOT successfully decreased the impedance of previously unusable small gold electrode sites to within viable neural recordings range. PEDOT coatings help to alleviate a major electrode design constraint, enabling the fabrication of ultrasmall, high-channel count arrays to interface chronically with the brain.

\section{Acknowledgments}

The authors would like to acknowledge all the members of the Neural Engineering Laboratory at the University of Michigan for their assistance in this study. This work was supported by the Center for Wireless Integrated Microsystems NSF EEC9986866, the NIH P41 Center for Neural Communication Technology (EB002030) and the Whitaker Foundation.

\section{References}

[1] Kipke D R et al 2008 Advanced neurotechnologies for chronic neural interfaces: new horizons and clinical opportunities J. Neurosci. 28 11830-8

[2] Bernatchez S F, Parks P J and Gibbons D F 1996 Interaction of macrophages with fibrous materials in vitro Biomaterials 17 2077-86 
[3] Sanders J E, Stiles C E and Hayes C L 2000 Tissue response to single-polymer fibers of varying diameters: evaluation of fibrous encapsulation and macrophage density J. Biomed. Mater. Res. 52 231-7

[4] Seymour J P and Kipke D R 2007 Neural probe design for reduced tissue encapsulation in CNS Biomaterials 28 3594-607

[5] Najafi K, Ji J and Wise K D 1990 Scaling limitations of silicon multichannel recording probes IEEE Trans. Biomed. Eng. $371-11$

[6] Kovacs G T A 1994 Enabling Technologies for Cultured Neural Networks ed D A Stenger and T McKenna (London: Academic) pp 121-65

[7] Schmidt E and Humphrey D R 1990 Neurophysiological Techniques vol 2 (Totowa, NJ: Humana Press) pp 1-64

[8] Shoham S and Nagarajan S 2003 Neuroprosthetics: Theory and Practice ed Kenneth W Horch and Gurpreet S Dhillon (Singapore: World Scientific) pp 448-65

[9] Robinson D A 1968 The electrical properties of metal microelectrodes Proc. IEEE 561065

[10] Hassibi A, Navid R, Dutton R W and Lee T H 2004 Comprehensive study of noise processes in electrode electrolyte interfaces J. Appl. Phys. 969

[11] Hetke J F, Lund J L, Najafi K, Wise K D and Anderson D J 1994 Silicon ribbon cables for chronically implantable microelectrode arrays IEEE Trans. Biomed. Eng. 41 314-21

[12] Ludwig K A, Uram J D, Yang J, Martin D C and Kipke D R 2006 Chronic neural recordings using silicon microelectrode arrays electrochemically deposited with a poly(3,4-ethylenedioxythiophene) (PEDOT) film J. Neural Eng. 359

[13] Cogan S F 2008 Neural stimulation and recording electrodes Annu. Rev. Biomed. Eng. 10 275-309

[14] Keefer E W, Botterman B R, Romero M I, Rossi A F and Gross G W 2008 Carbon nanotube coating improves neuronal recordings Nat. Nanotechnol. 3 434-9

[15] Abidian M R, Corey J M, Kipke D R and Martin D C 2010 Conducting-polymer nanotubes improve electrical properties, mechanical adhesion, neural attachment, and neurite outgrowth of neural electrodes Small 6 421-9

[16] Richardson-Burns S M et al 2007 Polymerization of the conducting polymer poly(3,4-ethylenedioxythiophene) (PEDOT) around living neural cells Biomaterials 28 1539-52

[17] Wilks S J, Richardson-Burns S M, Hendricks J L, Martin D C and Otto K J 2009 Poly(3,4-ethylenedioxythiophene) as a micro-neural interface material for electrostimulation Front Neuroeng. 27

[18] Cui X and Martin D C 2003 Electrochemical deposition and characterization of poly(3,4-ethylenedioxythiophene) on neural microelectrode arrays Sensors Actuators B 89 92-102

[19] Richardson-Burns S M, Hendricks J L and Martin D C 2007 Electrochemical polymerization of conducting polymers in living neural tissue J. Neural Eng. 4 L6

[20] Abidian M R and Martin D C 2008 Experimental and theoretical characterization of implantable neural microelectrodes modified with conducting polymer nanotubes Biomaterials 29 1273-83

[21] Abidian M R et al 2009 Interfacing conducting polymer nanotubes with the central nervous system: chronic neural recording using poly(3,4-ethylenedioxythiophene) nanotubes $A d v$. Mater. 218

[22] Vetter R J, Williams J C, Hetke J F, Nunamaker E A and Kipke D R 2004 Spike recording performance of implanted chronic silicon-substrate microelectrode arrays in cerebral cortex IEEE Trans. Neural Syst. Rehabil. Eng. 52 896-904

[23] Henze D A et al 2000 Intracellular features predicted by extracellular recordings in the hippocampus in vivo J. Neurophysiol. 84 390-400
[24] Yang J et al 2005 Ordered surfactant-templated poly(3,4-ethylenedioxythiophene) (PEDOT) conducting polymer on microfabricated neural probes Acta Biomater. 1 125-36

[25] Ludwig K A et al 2009 Using a common average reference to improve cortical neuron recordings from microelectrode arrays J. Neurophysiol. 101 1679-89

[26] Purcell E K, Thompson D E, Ludwig K A and Kipke D R 2009 Flavopiridol reduces the impedance of neural prostheses in vivo without affecting recording quality J. Neurosci. Methods

[27] Langhals N B and Kipke D R 2009 Validation of a novel three-dimensional electrode array within auditory cortex Conf. Proc. IEEE Eng. Med. Biol. Soc. 2009 2066-9

[28] Purcell E K, Thompson D E, Ludwig K A and Kipke D R 2009 Flavopiridol reduces the impedance of neural prostheses in vivo without affecting recording quality J. Neurosci. Methods 183 149-57

[29] Rohatgi P, Langhals N B, Kipke D R and Patil P G 2009 In vivo performance of a microelectrode neural probe with integrated drug delivery Neurosurg. Focus 27 E8

[30] Harris K D, Henze D A, Csicsvari J, Hirase H and Buzsaki G 2000 Accuracy of tetrode spike separation as determined by simultaneous intracellular and extracellular measurements J. Neurophysiol. 84 401-14

[31] Lewicki M S 1998 A review of methods for spike sorting: the detection and classification of neural action potentials Netw., Comput. Neural Syst. 9 R53-78

[32] Santhanam G et al 2007 HermesB: a continuous neural recording system for freely behaving primates IEEE Trans. Biomed. Eng. 54 2037-50

[33] Szarowski D H et al 2003 Brain responses to micro-machined silicon devices Brain Res. 983 23-35

[34] Turner J N et al 1999 Cerebral astrocyte response to micromachined silicon implants Exp. Neurol. 156 33-49

[35] Liu X et al 1999 Stability of the interface between neural tissue and chronically implanted intracortical microelectrodes IEEE Trans. Rehabil. Eng. 7 315-26

[36] Buitenweg J R, Rutten W L, Willems W P and van Nieuwkasteele J W 1998 Measurement of sealing resistance of cell-electrode interfaces in neuronal cultures using impedance spectroscopy Med. Biol. Eng. Comput. 36 630-7

[37] Grill W M and Mortimer J T 1994 Electrical properties of implant encapsulation tissue Ann. Biomed. Eng. 22 23-33

[38] Otto K J, Johnson M D and Kipke D R 2006 Voltage pulses change neural interface properties and improve unit recordings with chronically implanted microelectrodes IEEE Trans. Biomed. Eng. 53 333-40

[39] Williams J C, Hippensteel J A, Dilgen J, Shain W and Kipke D R 2007 Complex impedance spectroscopy for monitoring tissue responses to inserted neural implants $J$. Neural Eng. 4 410-23

[40] Vetter R J, Williams J C, Hetke J F, Nunamaker E A and Kipke D R 2004 Chronic neural recording using silicon-substrate microelectrode arrays implanted in cerebral cortex IEEE Trans. Biomed. Eng. 51 896-904

[41] Chen C S, Mrksich M, Huang S, Whitesides G M and Ingber D E 1997 Geometric control of cell life and death Science 276 1425-8

[42] Turner A M et al 2000 Attachment of astroglial cells to microfabricated pillar arrays of different geometries J. Biomed. Mater. Res. 51 430-41

[43] Biran R, Martin D C and Tresco P A 2005 Neuronal cell loss accompanies the brain tissue response to chronically implanted silicon microelectrode arrays Exp. Neurol. $195115-26$ 